Document downloaded from:

http://hdl.handle.net/10251/176384

This paper must be cited as:

López Alfonso, S. (2021). Vitali-Hahn-Saks property in coverings of sets algebras. Revista de la Real Academia de Ciencias Exactas Físicas y Naturales Serie A Matemáticas. 115(1):1-15. https://doi.org/10.1007/s13398-020-00952-x

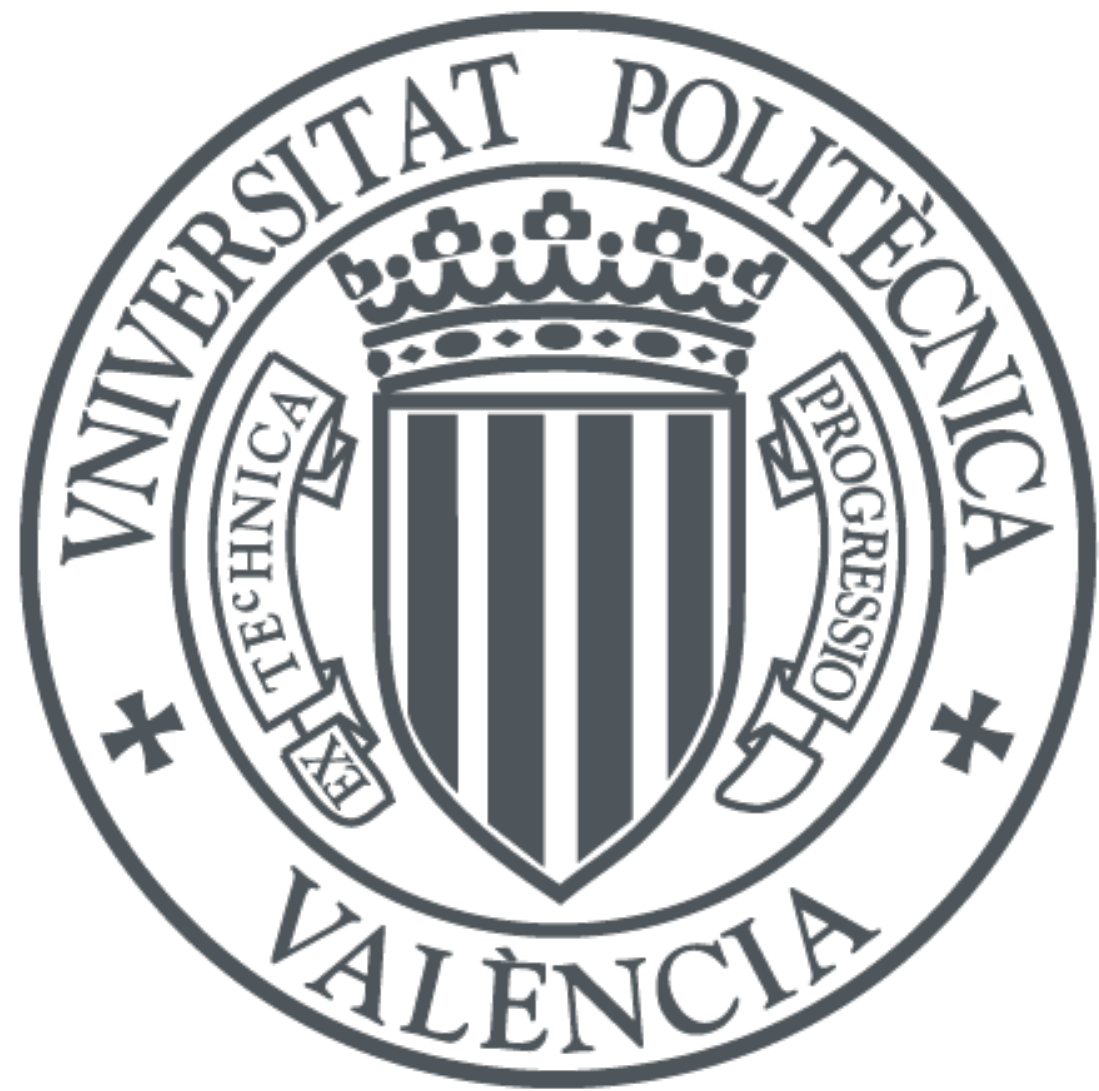

The final publication is available at

https://doi.org/10.1007/s13398-020-00952-x

Copyright Springer-Verlag

Additional Information 


\title{
Vitali-Hahn-Saks property in coverings of sets algebras
}

\section{S. López-Alfonso ${ }^{1}$}

Received: 25 September 2020 / Accepted: 14 October 2020

(c) The Royal Academy of Sciences, Madrid 2020

\begin{abstract}
A subset $\mathscr{B}$ of an algebra $\mathscr{A}$ of subsets of $\Omega$ is a Nikodým set for $b a(\mathscr{A})$ if each $\mathscr{B}$-pointwise bounded subset $M$ of $b a(\mathscr{A})$ is uniformly bounded on $\mathscr{A}$ and $\mathscr{B}$ is a strong Nikodým set for $b a(\mathscr{A})$ if each increasing covering $\left(\mathscr{B}_{m}\right)_{m=1}^{\infty}$ of $\mathscr{B}$ contains a $\mathscr{B}_{n}$ which is a Nikodým set for $b a(\mathscr{A})$, where $b a(\mathscr{A})$ is the Banach space of the real (or complex) finitely additive measures of bounded variation defined on $\mathscr{A}$. The subset $\mathscr{B}$ has $(V H S)$ property if $\mathscr{B}$ is a Nikodým set for $b a(\mathscr{A})$ and for each sequence $\left(\mu_{n}\right)_{m=1}^{\infty}$ and each $\mu$, both in $b a(\mathscr{A})$ and such that $\lim _{n \rightarrow \infty} \mu_{n}(B)=\mu(B)$, for each $B \in \mathscr{B}$, we have that the sequence $\left(\mu_{n}\right)_{m=1}^{\infty}$ converges weakly to $\mu$. We prove that if $\left(\mathscr{B}_{m}\right)_{m=1}^{\infty}$ is an increasing covering of and algebra $\mathscr{A}$ that has $(V H S)$ property and there exist a $\mathscr{B}_{n}$ which is a Nikodým set for $b a(\mathscr{A})$ then there exists $\mathscr{B}_{q}$, with $q \geq p$, such that $\mathscr{B}_{q}$ has $(V H S)$ property. In particular, if $\left(\mathscr{B}_{m}\right)_{m=1}^{\infty}$ is an increasing covering of a $\sigma$-algebra there exists $\mathscr{B}_{q}$ that has $(V H S)$ property. Valdivia proved that every $\sigma$-algebra has strong Nikodým property and in 2013 asked if Nikodým property in an algebra implies strong Nikodým property. We present three open questions related with this aforementioned Valdivia question and a proof of his strong Nikodým Theorem for $\sigma$ -algebras that it is independent of the Barrelled spaces theory and it is developed with basic results of Measure theory and Banach spaces.
\end{abstract}

Keywords Bounded set - Algebra and $\sigma$-algebra of subsets $\cdot$ Bounded finitely additive scalar measure $\cdot$ Nikodym and strong Nikodym property $\cdot$ Vitali-Hahn-Saks and strong Vitali-Hahn-Saks property

Mathematics Subject Classification 28A60 - 46G10

\section{Introduction}

Let $\mathscr{A}$ be an algebra of subsets of a set $\Omega$ and let $L(\mathscr{A})$ be the normed real or complex space generated by the characteristics functions $e(A)$ of the sets $A \in \mathscr{A}$ and endowed with the 25 supremum norm $\|\cdot\|_{\infty}$. Following [2, Theorem 1.13]) we identify its dual $L(\mathscr{A})^{*}$ provided with the dual norm isometrically with the Banach space ba $(\mathscr{A})$ of bounded finitely additive

\section{S. López-Alfonso}

salloal@csa.upv.es

1 Departamento Construcciones Arquitectónicas, Universitat Politècnica de València, 46022 Valencia, Spain 

the existence of an infinite dimensional separable quotient in a Banach space are presented ${ }_{58}$ in [12].

measures on $\mathscr{A}$ endowed with the variation norm, denoted by $|\cdot|(\Omega)$, or $|\cdot|$ in brief. Then for each $\mu \in \operatorname{ba}(\mathscr{A})$ and $C \in \mathscr{A}$ the value $\mu(C)$ represents both the value of the measure $\mu$ in $C$ and the value $\mu(e(C))$ of the linear form $\mu$ in $e(C)$. For an element $B$ of $\mathscr{A}$ the variation of $\mu$ on $B$ for each $\mu \in \mathrm{ba}(\mathscr{A})$ is named $|\mu|(B)$ and defines a seminorm in ba $(\mathscr{A})$ such that for each finite partition $\left\{B_{i}: B_{i} \in \mathscr{A}, 1 \leq i \leq n\right\}$ of $B$ we have $|\mu|(B)=\Sigma_{i}|\mu|\left(B_{i}\right)$.

Polar sets, named absolute polar sets in [9, Chapter IV, Sect. 20, 8 Polarity], are considered in the dual pair $<L(\mathscr{A})$, ba $(\mathscr{A})>$ and $M^{\circ}$ means the polar of a set $M$. If $\mathscr{B} \subset \mathscr{A}$ the topology $\tau_{S}(\mathscr{B})$ in ba $(\mathscr{A})$ is the topology of pointwise convergence in $\mathscr{B}$. In particular, $\tau_{S}(\mathscr{A})$ is the weak* topology in ba $(\mathscr{A})$ defined by the dual pair $\langle L(\mathscr{A})$, ba $(\mathscr{A})\rangle$.

The convex (absolutely convex) hull of a subset $M$ of a vector space is denoted by $\operatorname{co}(M)$ $(\operatorname{absco}(M))$. For a subset $B$ of a vector space $E$ the seminorm defined in span $B$ by $\inf \{|\lambda|$ : $x \in \lambda(\operatorname{absco} B)\}$, for each $x \in \operatorname{span} B$, is the gauge of absco $B$. The gauge of absco( $\left\{\chi_{C}: C \in\right.$ $\mathscr{A}\})$ is a norm in $L(\mathscr{A})$ with dual norm the $\mathscr{A}$-supremum norm, i.e., $\|\mu\|:=\sup \{|\mu(C)|$ : $C \in \mathscr{A}\}, \mu \in \operatorname{ba}(\mathscr{A})$. For $0<\alpha_{1} \leq \alpha_{2} \leq \cdots \leq \alpha_{m}$ and for a pairwise disjoint subsets $A_{i} \in \mathscr{A}, 1 \leq i \leq m$, the equalities

$$
\sum_{i=1}^{m} \alpha_{i} e\left(A_{i}\right)=\frac{\alpha_{1} e\left(\bigcup_{i=1}^{m} A_{i}\right)+\left(\alpha_{2}-\alpha_{1}\right) e\left(\bigcup_{i=2}^{m} A_{i}\right)+\cdots+\left(\alpha_{m}-\alpha_{m-1}\right) e\left(A_{m}\right)}{\alpha_{m}}
$$

and

$$
\frac{\alpha_{1}+\left(\alpha_{2}-\alpha_{1}\right)+\cdots+\left(\alpha_{m}-\alpha_{m-1}\right)}{\alpha_{m}}=1
$$

imply that the norms supremum $\|\cdot\|_{\infty}$ and the gauge of absco $\left(\left\{\chi_{C}: C \in \mathscr{A}\right\}\right)$ are equivalent, see [16, Propositions 1 and 2 for an inductive proof], hence its dual norms, variation in $\Omega$ and $\mathscr{A}$-supremum, are also equivalent. In general, for each $B \in \mathscr{A}$ the seminorms variation on $B$ and supremum of modulus on $\{C \in \mathscr{A}: C \subset B\}$ are equivalent seminorms in ba( $\mathscr{A})$.

If $\Sigma$ is a $\sigma$-algebra of subsets of a set $\Omega$ and $M$ is a $\Sigma$-pointwise bounded subset of ba( $\Sigma)$ then $M$ is a bounded subset of $\mathrm{ba}(\Sigma)$. We will refer this result as Nikodým boundedness theorem (see [1, page 80, named as Nikodym-Grothendieck Boundedness Theorem]). It is said that a subset $\mathscr{B}$ of an algebra $\mathscr{A}$ of subsets of a set $\Omega$ is a Nikodým set for ba( $\mathscr{A})$, or that $\mathscr{B}$ has property $(N)$ in brief, if each $\mathscr{B}$-pointwise bounded subset $M$ of $\mathrm{ba}(\mathscr{A})$ is bounded in $\mathrm{ba}(\mathscr{A})$, i.e., for a subset $M$ of ba $(\mathscr{A})$ the $\mathscr{B}$-pointwise boundedness is a deciding property for the uniform boundedness in the unit ball of $L(\mathscr{A})$ (see [15, Definition 2.4] or [17, Definition 56 1]). In the frame of uniform bounded deciding properties several equivalent results relative 57 to

Notice that in the definition of Nikodým set for ba $\mathscr{A})$ it is enough to consider that the subset $M$ is weak* closed and absolutely convex or that that $M$ is countable. Moreover, it is obvious that Nikodým boundedness theorem states that if $\Sigma$ is a $\sigma$-algebra then $\Sigma$ is a Nikodým set for ba $(\Sigma)$.

A subset $\mathscr{B}$ of an algebra $\mathscr{A}$ of subsets of a set $\Omega$ is a strong Nikodým set for ba( $\mathscr{A})$ if for each increasing covering $\left\{\mathscr{B}_{n}: n \in \mathbb{N}\right\}$ of $\mathscr{B}$ there exists $p \in \mathbb{N}$ such that $\mathscr{B}_{p}$ is a Nikodým set for ba $(\mathscr{A})$. The subset $\mathscr{B}$ is a web Nikodým set for ba $(\mathscr{A})$ if for each increasing web $\left\{\mathscr{B}_{n_{1} n_{2} \ldots n_{m}}: n_{i} \in \mathbb{N}, 1 \leq i \leq m, m \in \mathbb{N}\right\}$ of $\mathscr{B}$ there is a sequence $\left(p_{m}\right)_{m=1}^{\infty}$ such that $\mathscr{B}_{p_{1} p_{2} \ldots p_{m}}$ has $(N)$-property for every $m \in \mathbb{N}$. Remind that increasing web of $\mathscr{B}$ means that $\left\{\mathscr{B}_{n_{1}}: n_{1} \in \mathbb{N}\right\}$ is an increasing covering of $\mathscr{B}$ and that for each $m \in \mathbb{N}$ and for each $\left(n_{1} n_{2} \ldots n_{m}\right) \in \mathbb{N}^{m}$ we have that $\left\{\mathscr{B}_{n_{1} n_{2} \ldots n_{m} n_{m+1}}: n_{m+1} \in \mathbb{N}\right\}$ is an increasing covering of $\mathscr{B}_{n_{1} n_{2} \ldots n_{m}}$. In this paper algebra $(\sigma$-algebra) is used for algebra ( $\sigma$-algebra) of subsets of a set $\Omega$. 
Valdivia obtained in [16, Theorem 2] that for a $\sigma$-algebra $\Sigma$ the set $\Sigma$ is a strong Nikodým set for ba $(\Sigma)$ and in [17, Problem 1] he raised whether if for an algebra $\mathscr{A}$ it is true that the property $\mathscr{A}$ is a Nikodým set for ba $(\mathscr{A})$ implies that $\mathscr{A}$ is a strong Nikodým set for ba $\mathscr{A})$. This problem is still open and a partial solution has been obtained in [4, Theorem 3.3]. For a $\sigma$-algebra $\Sigma$ it was proved in [8, Theorem 2 ] and [11, Theorem 3] that $\Sigma$ is a web Nikodým set for ba $(\Sigma)$. Previous related results can be found in [5,14]. An example of an algebra $\mathscr{A}$ such that $\mathscr{A}$ is a web Nikodým set for ba $(\mathscr{A})$ is given in [10].

The completion of $L(\mathscr{A})$ endowed with the supremum norm $\|\cdot\|_{\infty}$ is the space $\widehat{L(\mathscr{A})}$ of bounded $\mathscr{A}$-measurable functions and an algebra of sets $\mathscr{A}$ has property $(G)$ if for each sequence $\left(\mu_{n}\right)_{n=1}^{\infty}$ of ba $(\mathscr{A})$ its weak* convergence to $\mu \in \mathrm{ba}(\mathscr{A})$, respect to the dual pair $\langle\widehat{L(\mathscr{A})}, \mathrm{ba}(\mathscr{A})\rangle$, implies its weak convergence to $\mu$, i.e.

$$
\lim _{n \rightarrow \infty} \mu_{n}(f)=\mu(f), \forall f \in \widehat{L(\mathscr{A})} \Rightarrow \lim _{n \rightarrow \infty} \mu_{n}(\varphi)=\mu(\varphi), \forall \varphi \in(\operatorname{ba}(\mathscr{A}))^{*},
$$

or, in brief, $\mathscr{A}$ has property $(G)$ if the space $\widehat{L(\mathscr{A})}$ is a Grothendieck space, see [15, Introduction] where it is stated that each $\sigma$-algebra has property $(G)$.

From Banach-Steinhaus theorem it follows that the condition $\lim _{n \rightarrow \infty} \mu_{n}(f)=\mu(f)$, for each $f \in \widehat{L(\mathscr{A})}$, implies that the sequence $\left(\mu_{n}\right)_{n=1}^{\infty}$ is bounded in ba $(\mathscr{A})$. Therefore an algebra of sets $\mathscr{A}$ has property $(G)$ if, and only if, each bounded sequence $\left(\mu_{n}\right)_{n=1}^{\infty}$ of ba $(\mathscr{A})$ such that $\lim _{n \rightarrow \infty} \mu_{n}(A)=\mu(A)$, for each $A \in \mathscr{A}$ with $\mu \in$ ba $(\mathscr{A})$, implies that the sequence $\left(\mu_{n}\right)_{n=1}^{\infty}$ converges weakly to $\mu$. This equivalence follows from the next straightforward Claim 1.

Claim 1 Let $F$ be a subset of a Banach space $E$ and let $\left(\mu_{n}\right)_{n=1}^{\infty}$ a bounded sequence in its dual $E^{*}$. If $\mu \in E^{*}$ and $\left(\mu_{n}\right)_{n=1}^{\infty}$ converges pointwise to $\mu$ in the subset $F$ then this sequence $\left(\mu_{n}\right)_{n=1}^{\infty}$ converges pointwise to $\mu$ in the closure $\bar{F}$ of $F$.

Proof In fact, let $\epsilon>0$ and $v \in \bar{F}$. By hypothesis there exists $f \in F$ such that $\|v-f\|<$ $\epsilon\left(2\left(1+|\mu|+\sup _{n}\left|\mu_{n}\right|\right)\right)^{-1}$, and for this $f$ there exists $n_{\epsilon}$ such that $\left|\left(\mu_{n}-\mu\right)(f)\right|<2^{-1} \epsilon$, for every $n>n_{\epsilon}$. Hence for $n>n_{\epsilon}$ we have that $\left|\left(\mu_{n}-\mu\right)(v)\right|$ is less than or equal than

$$
\left|\left(\mu_{n}-\mu\right)(v-f)\right|+\left|\left(\mu_{n}-\mu\right)(f)\right|<\frac{\epsilon\left(|\mu|+\sup _{n}\left|\mu_{n}\right|\right)}{2\left(1+|\mu|+\sup _{n}\left|\mu_{n}\right|\right.}+\frac{\epsilon}{2} \leq \epsilon,
$$

so $\left(\mu_{n}\right)_{n=1}^{\infty}$ converges pointwise to $\mu$ in $\bar{F}$.

A $\sigma$-algebra $\Sigma$ verifies the Vitali-Hahn-Saks theorem [15, Introduction]. This theorem states that every sequence $\left(\mu_{n}\right)_{n=1}^{\infty}$ of ba $(\Sigma)$ such that

$$
\lim _{n \rightarrow \infty} \mu_{n}(B)=\mu(B) \text {, for every } B \in \Sigma,
$$

is uniformly exhaustive, i.e., for each sequence $\left(B_{j}\right)_{j=1}^{\infty}$ of pairwise disjoint subsets of $\Sigma$ the $\lim _{j \rightarrow \infty} \mu_{n}\left(B_{j}\right)$ is 0 , uniformly in $n \in \mathbb{N}$. An algebra $\mathscr{A}$ has $(V H S)$ property if it verifies the thesis of Vitali-Hahn-Saks theorem and from [15, 2.5. Theorem], see also [7, Theorem 4.2], it follows that an algebra $\mathscr{A}$ has $(V H S)$ property if $\mathscr{A}$ has properties $(N)$ and $(G)$. Therefore $\mathscr{A}$ satisfies Vitali-Hahn-Saks $(V H S)$ property if and only $\mathscr{A}$ is a Nikodým set for ba $(\mathscr{A})$ and if for each sequence $\left(\mu_{n}\right)_{n=1}^{\infty}$ of ba( $\left.\mathscr{A}\right)$ and $\mu \in \mathrm{ba}(A)$ such that $\lim _{n \rightarrow \infty} \mu_{n}(A)=$ $\mu(A)$, for every $A \in \mathscr{A}$, we have that the sequence $\left(\mu_{n}\right)_{n=1}^{\infty}$ converges weakly to $\mu$. This characterization suggest the following definition. 
Definition 1 Let $\mathscr{B}$ a subset of an algebra $\mathscr{A}$. The subset $\mathscr{B}$ has $(V H S)$ property if $\mathscr{B}$ is a Nikodým set for ba $(A)$ and each sequence $\left(\mu_{n}\right)_{n=1}^{\infty}$ in ba $(\mathscr{A})$ and $\mu \in \mathrm{ba}(A)$ such that $\lim _{n \rightarrow \infty} \mu_{n}(B)=\mu(B)$, for each $B \in \mathscr{B}$, verify that $\left(\mu_{n}\right)_{n=1}^{\infty}$ converges weakly to $\mu$.

Vitali-Hahn-Saks theorem says that for a $\sigma$-algebra $\Sigma$ the set $\Sigma$ has (VHS) property. In the next section we prove that for each increasing covering $\left\{\mathscr{B}_{n}: n \in \mathbb{N}\right\}$ of $\Sigma$ there exists $p \in \mathbb{N}$ such that $\mathscr{B}_{p}$ has $(V H S)$ property and that for each increasing web $\left\{\mathscr{B}_{n_{1} n_{2} \ldots n_{m}}: n_{i} \in\right.$ $\mathbb{N}, 1 \leq i \leq m, m \in \mathbb{N}\}$ of $\Sigma$ there is a sequence $\left(p_{m}\right)_{m=1}^{\infty}$ such that $\mathscr{B}_{p_{1}} p_{2} \ldots p_{m}$ has $(V H S)$ property, for every $m \in \mathbb{N}$. We show that a positive solution of the mentioned Valdivia open problem [17, Problem 1] imply a positive solution for the corresponding problem for the $(V H S)$ property, i.e., that $(V H S)$ property for an algebra $\mathscr{A}$ implies strong $(V H S)$ property in $\mathscr{A}$, i.e., each increasing covering $\left\{\mathscr{A}_{n}: n \in \mathbb{N}\right\}$ of $\mathscr{A}$ contains an $\mathscr{A}_{p}$ with the $(V H S)$ property.

In the last section we provide a proof of Valdivia theorem stating that for each $\sigma$-algebra $\Sigma$ the set $\Sigma$ has the strong Nikodým property. This proof is dedicated to M. Valdivia, follows Valdivia's scheme in [16], it is made with basic elements of measure theory and a few elementary properties of Banach spaces. Therefore it is independent of the theory of barrelled spaces and it may help researchers interested in this subject and not familiar with barrelled spaces. Barrelled spaces are locally convex spaces that verify the Banach-Steinhaus theorem and its main properties may be found in $[3,6,13]$, among others.

\section{Sets with (VHS) property}

Proposition 1 gives a characterization of Nikodým sets for ba $(\mathscr{A})$.

Proposition 1 A subset $\mathscr{B}$ of an algebra of sets $\mathscr{A}$ is a Nikodým set for ba( $\mathscr{A})$ if and only if for each increasing covering $\left\{\mathscr{B}_{n}: n \in \mathbb{N}\right\}$ of $\mathscr{B}$ there exists $p \in \mathbb{N}$ such that

$$
\overline{\operatorname{absco}\left\{e(A): A \in \mathscr{B}_{p}\right\}} L(\mathscr{A})
$$

is a neighborhood of zero in $L(\mathscr{A})$.

Hence $\overline{\operatorname{span}\{e(A): A \in \mathscr{B} p}^{L(\mathscr{A})}=L(\mathscr{A})$ and $\overline{\operatorname{absco}\{e(A): A \in \mathscr{B} p}^{\widehat{L(\mathscr{A})}}$ is a neighborhood of zero in $\widehat{L(\mathscr{A})}$.

Proof If $\mathscr{B}$ is a not a Nikodým set for ba( $\mathscr{A})$ there exists an unbounded subset $C$ in ba $(\mathscr{A})$ which is pointwise bounded in $\mathscr{B}$. This implies that the family of sets $\mathscr{B}_{n}=\{A \in \mathscr{B}$ : $\left.\sup _{\mu \in C}|\mu(A)| \leq n\right\}, n \in \mathbb{N}$, are an increasing covering of $\mathscr{B}$ such that $\left\{e(A): A \in \mathscr{B}_{n}\right\} \subset$ $n C^{\circ}$, for each $n \in \mathbb{N}$, hence

$$
{\overline{\operatorname{absco}\left\{e(A): A \in \mathscr{B}_{n}\right\}}}^{L(\mathscr{A})} \subset n C^{\circ} .
$$

As $C$ is an unbounded subset of ba $(\mathscr{A})$ we have that $n C^{\circ}$ is not a neighborhood of zero in

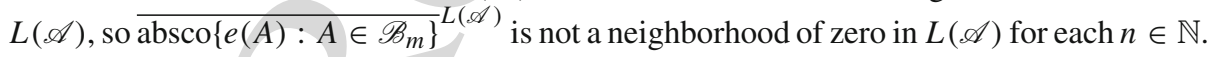
If there exists an increasing covering $\left\{\mathscr{B}_{n}: n \in \mathbb{N}\right\}$ of $\mathscr{B}$ such that

$$
\overline{\operatorname{absco}\left\{e(A): A \in \mathscr{B}_{n}\right\}} L(\mathscr{A})
$$

is not a neighborhood of zero in $L(\mathscr{A})$ for every $n \in \mathbb{N}$, then the polar sets $\left\{e(A): A \in \mathscr{B}_{n}\right\}^{\circ}$ are unbounded, so there exists $\mu_{n} \in\left\{e(A): A \in \mathscr{B}_{n}\right\}^{\circ}$ such that $\left|\mu_{n}\right| \geq n$, for each $n \in \mathbb{N}$. If $A \in \mathscr{B}$ there exists $q_{A} \in \mathbb{N}$ such that $A \in \mathscr{B}_{n}$ for each $n \geq q_{A}$, hence $\left|\mu_{n}(e(A))\right| \leq 1$ 
for $n \geq q_{A}$, and we get that $\left\{\left|\mu_{n}(e(A))\right|: n \in \mathbb{N}\right\}$ is $\tau_{s}(\mathscr{B})$-bounded, hence $\mathscr{B}$ is a not a Nikodým set for ba $(\mathscr{A})$.

In particular, if $\mathscr{B}$ is a Nikodým set for ba $(\mathscr{A})$ then $\overline{\operatorname{absco}\{e(A): A \in \mathscr{B}\}}^{L(\mathscr{A})}$ is a neighborhood of zero in $L(\mathscr{A})$ and $\overline{\operatorname{span}\{e(A): A \in \mathscr{B}\}}^{L(\mathscr{A})}=L(\mathscr{A})$.

It is said that an increasing web $\left\{\mathscr{C}_{n_{1} n_{2} \ldots n_{m}}: n_{i} \in \mathbb{N}, 1 \leq i \leq m, m \in \mathbb{N}\right\}$ of $\mathscr{B}$ is contained in the increasing web $\left\{\mathscr{B}_{n_{1} n_{2} \ldots n_{m}}: n_{i} \in \mathbb{N}, 1 \leq i \leq m, m \in \mathbb{N}\right\}$ of $\mathscr{B}$ if for each sequence $\left(q_{m}\right)_{m=1}^{\infty}$ of natural numbers there exists a sequence $\left(p_{m}\right)_{m=1}^{\infty}$ of natural numbers such that $q_{m} \leq p_{m}$ and $\mathscr{C}_{q_{1} q_{2} \ldots q_{m}}=\mathscr{B}_{p_{1} p_{2} \ldots p_{m}}$, for each $m \in \mathbb{N}$.

Corollary 1 Let $\mathscr{A}$ be an algebra of sets with a subset $\mathscr{B}$ that it is a web Nikodým set for ba $(\mathscr{A})$. Each increasing web $\left\{\mathscr{B}_{n_{1} n_{2} \ldots n_{m}}: n_{i} \in \mathbb{N}, 1 \leq i \leq m, m \in \mathbb{N}\right\}$ of $\mathscr{B}$ contains and increasing web $\left\{\mathscr{C}_{n_{1} n_{2} \ldots n_{m}}: n_{i} \in \mathbb{N}, 1 \leq i \leq m, m \in \mathbb{N}\right\}$ of $\mathscr{B}$ such that each $\mathscr{C}_{n_{1} n_{2} \ldots n_{m}}$ is a Nikodým set for $\mathrm{ba}(\mathscr{A})$ and $\overline{\mathrm{absco}\left\{e(A): A \in \mathscr{C}_{n_{1} n_{2} \ldots n_{m}}\right\}}{ }^{L(\mathscr{A})}$ is a neighborhood of zero in $L(\mathscr{A})$.

Proof By contradiction we get easily that if $\mathscr{B}$ is a web Nikodym set for ba $(\mathscr{A})$ then if for each increasing covering $\left\{\mathscr{B}_{n}: n \in \mathbb{N}\right\}$ of $\mathscr{B}$ there exists $p_{1} \in \mathbb{N}$ such that for each $n \geq p_{1}$ the set $\mathscr{B}_{n}$ is also a web Nikodým set for ba $(\mathscr{A})$. Additionally, by Proposition 1 there exists $p \in \mathbb{N}, p \geq p_{1}$, such that ${\overline{\operatorname{absco}\left\{e(A): A \in \mathscr{B}_{n}\right\}}}^{L(\mathscr{A})}$ is a neighborhood of zero in $L(\mathscr{A})$, for each $n \geq p$. The Corollary follows by a trivial induction.

Problem 1 Let $\left\{\mathscr{A}_{n}: n \in \mathbb{N}\right\}$ be an increasing covering of an algebra $\mathscr{A}$ with (VHS) property. We do not know if there exists a natural number $p$ such that $\mathscr{A}_{p}$ has (VHS) property.

Proposition 2 shows that a total or partial positive solution of mentioned Valdivia open Problem [17, Problem 1] implies a total or partial positive solution of Problem 1.

Proposition 2 Let $\left\{\mathscr{A}_{n}: n \in \mathbb{N}\right\}$ be an increasing covering of an algebra $\mathscr{A}$ with $(V H S)$ property. If there exists $p$ such that $\mathscr{A}_{p}$ is a Nikodym set for ba $(A)$ then there exists $q \in \mathbb{N}$ such that $\mathscr{A}_{q}$ has the $(V H S)$ property.

Proof $\mathscr{A}$ and $\mathscr{A}_{n}, n \geq p$, are Nikodým sets for ba $(A)$, hence by Proposition 1 there exists $q \geq p$ such that $\mathscr{A}_{q}$ is a Nikodým set for ba $(\Sigma)$ and $\overline{\operatorname{absco}\left\{e(A): A \in \mathscr{A}_{q}\right\}} \widehat{L(\Sigma)}$ is a neighborhood of 0 in $\widehat{L(\mathscr{A})}$. Let $\left(\mu_{n}\right)_{n=1}^{\infty}$ be a sequence in ba $(\mathscr{A})$ and $\mu \in \mathrm{ba}(\mathscr{A})$ such that $\lim _{n \rightarrow \infty} \mu_{n}(B)=\mu(B)$, for each $B \in \mathscr{A}_{q}$. It is obvious that $\lim _{n \rightarrow \infty} \mu_{n}(f)=\mu(f)$, for each $f \in \operatorname{absco}\left\{e(A): A \in \mathscr{A}_{q}\right\}$.

As $\mathscr{A}_{q}$ is a Nikodým set for ba( $\left.\mathscr{A}\right)$ then $\left\{\mu_{n}: n \in \mathbb{N}\right\}$ is a bounded subset of ba $(\mathscr{A})$. Then Claim 1 implies that $\lim _{n \rightarrow \infty} \mu_{n}(f)=\mu(f)$, for each $f \in{\overline{\operatorname{absco}\left\{e(A): A \in \mathscr{A}_{q}\right\}}}^{\widehat{L(\Sigma)}}$, so also $\lim _{n \rightarrow \infty} \mu_{n}(f)=\mu(f)$, for each $f \in \widehat{L(\mathscr{A})}$. From this property and the hypothesis that $\mathscr{A}$ has property $(G)$, it follows that $\left(\mu_{n}\right)_{n=1}^{\infty}$ converges weakly to $\mu$, i.e., $\lim _{n \rightarrow \infty} \mu_{n}(f)=$ $\mu(f)$ for each $f \in(\mathrm{ba}(\mathscr{A}))^{*}$, hence $\mathscr{A}_{q}$ has $(V H S)$ property.

In particular, by [16, Theorem 2] and [15, Introduction] it follows that if $\left(\mathscr{B}_{n}\right)_{n=1}^{\infty}$ is an increasing covering of a $\sigma$-algebra $\Sigma$ there exists $p \in \mathbb{N}$ such that $\mathscr{B}_{p}$ has (VHS) property. This result is a particular case of the following Theorem. 
Theorem 1 Let $\left\{\mathscr{B}_{n_{1} n_{2} \ldots n_{m}}: n_{i} \in \mathbb{N}, 1 \leq i \leq m, m \in \mathbb{N}\right\}$ be an increasing web of a $\sigma$-algebra $\Sigma$. There exists an increasing web $\left\{\mathscr{C}_{n_{1} n_{2} \ldots n_{m}}: n_{i} \in \mathbb{N}, 1 \leq i \leq m, m \in \mathbb{N}\right\}$ contained in $\left\{\mathscr{B}_{n_{1} n_{2} \ldots n_{m}}: n_{i} \in \mathbb{N}, 1 \leq i \leq m, m \in \mathbb{N}\right\}$ such that each $\mathscr{C}_{n_{1} n_{2} \ldots n_{m}}$ has (VHS) property for every $\left(n_{1} n_{2} \ldots n_{m}\right) \in \mathbb{N}^{m}$ and $m \in \mathbb{N}$.

Proof By [8, Theorem 2] and [11, Theorem 3] $\Sigma$ is a web Nikodým set for ba( $\mathscr{A})$. By Corollary 1 there exists an increasing web $\left\{\mathscr{C}_{n_{1} n_{2} \ldots n_{m}}: n_{i} \in \mathbb{N}, 1 \leq i \leq m, m \in \mathbb{N}\right\}$ contained in $\left\{\mathscr{B}_{n_{1} n_{2} \ldots n_{m}}: n_{i} \in \mathbb{N}, 1 \leq i \leq m, m \in \mathbb{N}\right\}$ such that each $\mathscr{C}_{n_{1} n_{2} \ldots n_{m}}$ is a Nikodým set for ba $(\Sigma)$ and ${\overline{\operatorname{absco}\left\{e(A): A \in \mathscr{C}_{n_{1} n_{2} \ldots n_{m}}\right\}}}^{L(\mathscr{A})}$ is a neighborhood of zero in $L(\Sigma)$. Let $\left(\mu_{n}\right)_{n=1}^{\infty}$ be a sequence in $\mathrm{ba}(\Sigma)$ and $\mu \in \mathrm{ba}(\Sigma)$ such that $\lim _{n \rightarrow \infty} \mu_{n}(B)=$ $\mu(B)$, for each $B \in \mathscr{C}_{n_{1} n_{2} \ldots n_{m}}$. It is obvious that $\lim _{n \rightarrow \infty} \mu_{n}(f)=\mu(f)$, for each $f \in$ $\operatorname{absco}\left\{e(A): A \in \mathscr{C}_{n_{1} n_{2} \ldots n_{m}}\right\}$.

As $\mathscr{C}_{n_{1} n_{2} \ldots n_{m}}$ is a Nikodým set for ba(A) we get that $\left\{\mu_{n}: n \in \mathbb{N}\right\}$ is a bounded subset of ba( $\mathscr{A})$. Claim 1 imply $\lim _{n \rightarrow \infty} \mu_{n}(f)=\mu(f)$, for each $f \in$ $\overline{\operatorname{absco}\left\{e(A): A \in \mathscr{C}_{n_{1} n_{2} \ldots n_{m}}\right\}} \widehat{\widehat{L(\Sigma)}}$, so also $\lim _{n \rightarrow \infty} \mu_{n}(f)=\mu(f)$, for each $f \in \widehat{L(\Sigma)}$. From this property and the fact that every $\sigma$-algebra has property $(G)$, see $[15$, Introduction], it follows that $\left(\mu_{n}\right)_{n=1}^{\infty}$ converges weakly to $\mu$, i.e., $\lim _{n \rightarrow \infty} \mu_{n}(f)=\mu(f)$ for each $f \in(\operatorname{ba}(\mathscr{A}))^{*}$, hence $\mathscr{C}_{n_{1} n_{2} \ldots n_{m}}$ has $(V H S)$ property.

\section{Revisiting Valdivia theorem on Nikodým sets}

In this section we provide a proof of Valdivia theorem stating that for each $\sigma$-algebra $\Sigma$ the set $\Sigma$ is a strong Nikodým set for ba $(\Sigma)$, see Theorem 2 . This proof only needs basic results of Measure theory and Banach spaces.

The main results of this section are Propositions 3 and 4. Both are preceded by several Claims and Lemmas to help its reading. An induction based in Proposition 4 gives Proposition 5 and a countable subset of the sets and measures obtained in Proposition 5 enables to prove Valdivia theorem on Nikodým sets in Theorem 2.

Claim 2 Let $B$ and $C$ be two subsets of a vector space $E$. If $C$ is finite there exists a subset $D$ of $C$ such that $\operatorname{span} B \cap \operatorname{span} D=\{0\}$ and the gauges defined by $\operatorname{absco}(B \cup C)$ and $\operatorname{absco}(B \cup D)$ are equivalents.

Proof If $\operatorname{span} B \cap \operatorname{span} C \neq\{0\}$ then there exists $q_{1}=\sum_{i=1}^{p} \beta_{i} b_{i}+\sum_{j=1}^{q} \gamma_{j} c_{j} \in C \backslash\{0\}$, with each $\left(b_{i}, c_{j}\right) \in B \times\left(C \backslash\left\{q_{1}\right\}\right)$. If $x \in \operatorname{absco}(B \cup C)$ then $x=\sum_{i=1}^{s} \delta_{i} d_{i}+\epsilon q_{1}+\sum_{j=1}^{t} \epsilon_{j} e_{j}$, with $\sum_{i=1}^{s}\left|\delta_{i}\right|+|\epsilon|+\sum_{j=1}^{t}\left|\epsilon_{j}\right| \leq 1$ and $\left(d_{i}, e_{j}\right) \in B \times\left(C \backslash\left\{q_{1}\right\}\right)$, therefore

$$
x=\sum_{i=1}^{s} \delta_{i} d_{i}+\sum_{i=1}^{p} \epsilon \beta_{i} b_{i}+\sum_{j=1}^{q} \epsilon \gamma_{j} c_{j}+\sum_{j=1}^{t} \epsilon_{j} e_{j} .
$$

If $h=\sum_{i=1}^{p}\left|\beta_{i}\right|+\sum_{j=1}^{q}\left|\gamma_{j}\right|$ then the inequality

$$
\sum_{i=1}^{s}\left|\delta_{i}\right|+\sum_{i=1}^{p}\left|\epsilon \beta_{i}\right|+\sum_{j=1}^{q}\left|\epsilon \gamma_{j}\right|+\sum_{j=1}^{t}\left|\epsilon_{j}\right| \leq 1+h,
$$


provides the second inclusion in

$$
\operatorname{absco}\left(B \cup\left(C \backslash\left\{q_{2}\right\}\right)\right) \subset \operatorname{absco}(B \cup C) \subset(1+h) \operatorname{absco}\left(B \cup\left(C \backslash\left\{q_{1}\right\}\right)\right) .
$$

The first inclusion in (1) is obvious and (1) implies that the gauges defined by the sets $\operatorname{absco}(B \cup C)$ and $\operatorname{absco}\left(B \cup C \backslash\left\{q_{1}\right\}\right)$ are equivalents. If $\operatorname{span} B \cap \operatorname{span}\left(C \backslash\left\{q_{1}\right\}\right) \neq\{0\}$ then with the previous construction we determine a vector $q_{2} \in C \backslash\left\{q_{1}\right\}$ such that the gauges defined by $\operatorname{absco}\left(B \cup C \backslash\left\{q_{1}\right\}\right)$ and by $\operatorname{absco}\left(B \cup C \backslash\left\{q_{1}, q_{2}\right\}\right)$ are equivalents. After a finite number $r$ of repetitions of this process we get a finite subset $D=C \backslash\left\{q_{1}, q_{2}, \ldots, q_{r}\right\}$ such that gauges defined by $\operatorname{absco}(B \cup C)$ and by $\operatorname{absco}(B \cup D)$ are equivalents and $\operatorname{span} B \cap \operatorname{span} D=\{0\}$. This proves the Claim.

If $F$ is a dense subspace of a normed space $E, x \in E$ and $0<\|x\|<r$ then there exists a sequence $\left(x_{n}\right)_{n=1}^{\infty}$ in $F$ such that $\left\|x_{n}\right\|<r, n \in \mathbb{N}$, and $\lim _{n \rightarrow \infty} x_{n}=x$. Therefore

$$
\overline{\{x \in F:\|x\|<r\}}^{E}=\{x \in E:\|x\| \leq r\} .
$$

In particular, if $B$ is a zero neighborhood in span $B$ and $\overline{\operatorname{span} B}^{E}=E$ then $\bar{B}^{E}$ is a neighborhood of the null vector of $E$. This observation is used in the following claim.

Claim 3 Let $B$ be a closed absolutely convex subset of the normed space $E$ such that $\overline{\operatorname{span}} B^{E}=E$. If $B$ is not a zero neighborhood in $E$ then for each finite subset $C$ of $E$ we have that

$$
\operatorname{absco}(B \cup C)
$$

is not a zero neighborhood in E.

Proof By Claim 2 there exists a finite subset $D$ in $C$ such that the gauges of $\operatorname{absco}(B \cup C)$ and $\operatorname{absco}(B \cup D)$ are equivalent and the algebraic sum $\operatorname{span} B+\operatorname{span} D$ is direct. Hence if $\operatorname{absco}(B \cup C)$ is a zero neigbourhood in $E$ then, by equivalence, $\operatorname{absco}(B \cup D)$ is also a zero neighborhood in $E$ and then $(\operatorname{absco}(B \cup D) \cap(\operatorname{span} B)$ would be a neighborhood of zero in span $B$. As the algebraic sum span $B+\operatorname{span} D$ is direct we have that

$$
(\operatorname{absco}(B \cup D)) \cap(\operatorname{span} B)=B,
$$

and we get that $B$ is zero neighborhood in span $B$. The condition $\overline{\operatorname{span}}^{E}=E$ imply that the closed set $B=\bar{B}$ is a neighborhood of zero in $E$. From this contradiction follows the proposition.

Lemma 1 Let $M$ be an unbounded, weak ${ }^{*}$-closed and absolutely convex subset of ba( $\left.\mathscr{A}\right)$ such that ${\overline{\operatorname{span}} M^{\circ}}^{L(\mathscr{A})}=L(\mathscr{A})$. For each finite subset $\mathrm{Q}$ of $\mathscr{A}$ we have that $M \cap\{e(A): A \in \mathrm{Q}\}^{\circ}$ is unbounded in ba $(\mathscr{A})$, i.e.,

$$
\sup _{\mu \in M \cap\{e(A): A \in Q\}^{\circ}}\{|\mu|(\Omega)\}=\infty .
$$

Proof The set $B=M^{\circ}$ verifies the conditions in Claim 3. So the set $\operatorname{absco}(B \cup\{e(A): A \in$ $Q\})$ is not a zero neighborhood in $L(\mathscr{A})$. Hence its polar set

$$
\{\operatorname{absco}(B \cup\{e(A): A \in Q\})\}^{\circ}=M^{\circ \circ} \cap\{e(A): A \in Q\}^{\circ}
$$

is an unbounded subset of $\mathrm{ba}(\mathscr{A})$ and as $M=M^{\circ \circ}$ we get (3). 
Proposition 3 Let $\left\{\mathscr{B}_{n}: n \in \mathbb{N}\right\}$ be an increasing covering of a subset of $\mathscr{B}$ of an algebra $\mathscr{A}$. If $\mathscr{B}$ is a Nikodým set for ba $\mathscr{A})$ and for every $n \in \mathbb{N}$ the set $\mathscr{B}_{n}$ is not a Nikodým for ba( $\left.\mathscr{A}\right)$, then there exists $p \in \mathbb{N}$ such that for each $n \geq p$ there exists a subset $M_{n}$ in ba( $\left.\mathscr{A}\right)$ that it is $\mathscr{B}_{n}$-pointwise bounded, absolutely convex, weak ${ }^{*}$-closed and such that for each finite subset $\mathrm{Q}$ of $\mathscr{A}$ the intersection $M_{n} \cap\{e(A): A \in \mathrm{Q}\}^{\circ}$ is unbounded in ba $(\mathscr{A})$.

Proof By Proposition 1 there exists $p \in \mathbb{N}$ such that for each $n \geq p$

$$
{\overline{\operatorname{span}\left\{e(A): A \in \mathscr{B}_{n}\right\}}}^{L(\mathscr{A})}=L(\mathscr{A}) .
$$

As $\mathscr{B}_{n}$ is not a Nikodým for ba $(\mathscr{A})$ there exists an unbounded, weak*-closed and absolutely

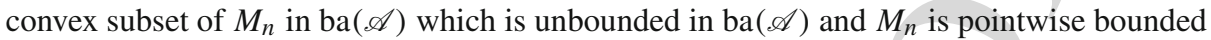
in $\left\{e(A): A \in \mathscr{B}_{n}\right\}$. The pointwise boundedness imply that $\left\{e(A): A \in \mathscr{B}_{n}\right\} \subset \operatorname{span} M_{n}^{\circ}$, hence for each $n \geq p$ we have by (4) that

$$
L(\mathscr{A})={\overline{\operatorname{span}\left\{e(A): A \in \mathscr{B}_{n}\right\}}}^{L(\mathscr{A})} \subset{\overline{\operatorname{span} M_{n}^{\circ}}}^{L(\mathscr{A})} \subset L(\mathscr{A}) .
$$

From (5) we deduce that $\operatorname{span} \bar{M}_{n}^{\circ} L(\mathscr{A})=L(\mathscr{A})$, for each $n \geq p$, and the Proposition follows from Lemma 1.

Claim 4 Let $B$ be an element of an algebra $\mathscr{A}$, let $M$ be a subset of ba $(\mathscr{A})$ such that for each finite subset $\mathrm{Q}$ of $\mathscr{A}$

$$
\sup _{\mu \in M \cap\{e(A): A \in Q\}^{\circ}}\{|\mu|(B)\}=\infty
$$

if $\left\{B_{1}, B_{2}, \ldots, B_{q}\right\}$ is a finite partition of $B$ by elements of $\mathscr{A}$ there exist $j, 1 \leq j \leq q$, such that for each finite subset $\mathrm{Q}$ of $\mathscr{A}$

$$
\sup _{\mu \in M \cap\left\{e(A): A \in \mathrm{Q}^{\circ}\right.}\left\{|\mu|\left(B_{j}\right)\right\}=\infty .
$$

Proof The first member of (6) is equal to

$$
\sum_{i=1}^{q} \sup _{\mu \in M \cap\{e(A): A \in Q\}^{\circ}}\left\{|\mu|\left(B_{i}\right)\right\}
$$

that with (6) implies (7).

The next Claim 5 will used in Lemma 2.

Claim 5 Let $\mathscr{A}$ be an algebra of subsets of a set $\Omega, A \in \mathscr{A}$ and $M$ a weak*-closed and absolutely convex subset of $\mathrm{ba}(\mathscr{A})$ such that for each finite subset $\mathrm{Q}$ of $\mathscr{A}$

$$
\sup _{\mu \in M \cap\{e(D): D \in \mathrm{Q}\}^{\circ}}|\mu|(A)=\infty .
$$

Then for each $\alpha \in \mathbb{R}^{+}$and each subset $\left\{B_{i}: 1 \leq i \leq n\right\}$ of $\mathscr{A}$ there exists $\left(\mu_{1}, A_{1}\right) \in M \times \mathscr{A}$, $A_{1} \subset$ A such that

$$
\left|\mu_{1}\left(e\left(A_{1}\right)\right)\right|>\alpha,\left|\mu_{1}\left(e\left(A \backslash A_{1}\right)\right)\right|>\alpha, \sum_{j=1}^{n}\left|\mu_{1}\left(e\left(B_{j}\right)\right)\right| \leq 1
$$

and for each finite subset $\mathrm{Q}$ of $\mathscr{A}$

$$
\sup _{\mu \in M \cap\{e(D): D \in \mathrm{Q}\}^{\circ}}|\mu|\left(A \backslash A_{1}\right)=\infty .
$$


Proof By (8) with $Q=\left\{A, B_{1}, \ldots, B_{n}\right\}$ there exists $\left(v_{1}, P_{11}\right) \in\left(M \cap\{e(D): D \in Q\}^{\circ}\right) \times \mathscr{A}$, with $P_{11} \subset A$ such that

$$
\left|v_{1}\left(P_{11}\right)\right|>n(\alpha+1),\left|v_{1}(A)\right| \leq 1 \text { and }\left|v_{1}\left(B_{j}\right)\right| \leq 1 \text {, for } 1 \leq j \leq n .
$$

Let $P_{12}:=A \backslash P_{11}$ and $\mu_{1}=n^{-1} v_{1}$. The measure $\mu_{1} \in M$ and verifies that

$$
\left|\mu_{1}\left(P_{11}\right)\right|>\alpha+1,\left|\mu_{1}(A)\right| \leq 1, \sum_{j=1}^{n}\left|\mu_{1}\left(e\left(B_{j}\right)\right)\right| \leq 1,
$$

hence

$$
\left|\mu_{1}\left(P_{12}\right)\right|=\left|\mu_{1}(A)-\mu_{1}\left(P_{11}\right)\right| \geq\left|\mu_{1}\left(P_{11}\right)\right|-\left|\mu_{1}(A)\right|>\alpha .
$$

By Claim 4 it is verified at least one of the inequalities

$$
\sup _{\mu \in M \cap\{e(D): D \in Q\}^{\circ}}\left\{|\mu|\left(P_{11}\right)\right\}=\infty \text {, for each finite subset } Q \in \mathscr{A}
$$

or

$$
\sup _{\mu \in M \cap\{e(D): D \in Q\}^{\circ}}\left\{|\mu|\left(P_{12}\right)\right\}=\infty \text {, for each finite subset } Q \in \mathscr{A}
$$

In the first we define $A_{1}:=P_{12}$ and in the second we take $A_{1}:=P_{11}$ to get this Claim.

Lemma 2 Let $\mathscr{A}$ be an algebra of subsets of a set $\Omega, A \in \mathscr{A}$ and $M$ a weak*-closed and absolutely convex subset of ba( $\mathscr{A})$ such that for each finite subset $\mathrm{Q}$ of $\mathscr{A}$

$$
\sup _{\mu \in M \cap\{e(D): D \in \mathrm{Q}\}^{\circ}}|\mu|(A)=\infty .
$$

For each $(p, \alpha) \in(\mathbb{N} \backslash\{0,1\}) \times \mathbb{R}^{+}$and each finite subset $\left\{B_{i}: 1 \leq i \leq n\right\}$ of $\mathscr{A}$ there exists a partition $\left\{A_{i}: A_{i} \in \mathscr{A}, 1 \leq i \leq p\right\}$ of $A$ and a subset $\left\{\mu_{i}: 1 \leq i \leq p\right\}$ of $M$ such that

$$
\left|\mu_{i}\left(e\left(A_{i}\right)\right)\right|>\alpha \text { and } \sum_{j=1}^{n}\left|\mu_{i}\left(e\left(B_{j}\right)\right)\right| \leq 1, \text { for } 1 \leq i \leq p
$$

Proof By Claim 5 there exists in $A$ a partition $\left\{A_{1}, A \backslash A_{1}\right\} \in \mathscr{A} \times \mathscr{A}$ and a measure $\mu_{1} \in M$ such that

$$
\left|\mu_{1}\left(e\left(A_{1}\right)\right)\right|>\alpha,\left|\mu_{1}\left(e\left(A \backslash A_{1}\right)\right)\right|>\alpha, \sum_{j=1}^{n}\left|\mu_{1}\left(e\left(B_{j}\right)\right)\right| \leq 1
$$

and for each finite subset $Q$ of $\mathscr{A}$

$$
\sup _{\mu \in M \cap\{(D): D \in Q\}^{\circ}}|\mu|\left(A \backslash A_{1}\right)=\infty .
$$

If we apply to $A \backslash A_{1}$ the Claim 5 we get in $A \backslash A_{1}$ a partition $\left\{A_{2}, A \backslash\left(A_{1} \cup A_{2}\right)\right\} \in \mathscr{A} \times \mathscr{A}$ and a measure $\mu_{2} \in M$ such that

$$
\left|\mu_{2}\left(e\left(A_{2}\right)\right)\right|>\alpha,\left|\mu_{2}\left(e\left(A \backslash\left(A_{1} \cup A_{2}\right)\right)\right)\right|>\alpha, \sum_{j=1}^{n}\left|\mu_{2}\left(e\left(B_{j}\right)\right)\right| \leq 1
$$

and for each finite subset $Q$ of $\mathscr{A}$

$$
\sup _{\mu \in M \cap\{e(D): D \in Q\}^{\circ}}|\mu|\left(A \backslash\left(A_{1} \cup A_{2}\right)\right)=\infty .
$$


Following this method we get in $A \backslash\left(A_{1} \cup A_{2} \cup \cdots \cup A_{p-2}\right)$ a partition $\left\{A_{p-1}, A \backslash\left(A_{1} \cup\right.\right.$ $\left.\left.A_{2} \cup \cdots \cup A_{p-2} \cup A_{p-1}\right)\right\} \in \mathscr{A} \times \mathscr{A}$ and a measure $\mu_{p-1} \in M$ such that $\left|\mu_{p-1}\left(e\left(A_{p-1}\right)\right)\right|>\alpha,\left|\mu_{p-1}\left(e\left(A \backslash\left(A_{1} \cup \cdots \cup A_{p-1}\right)\right)\right)\right|>\alpha, \sum_{j=1}^{n}\left|\mu_{p-1}\left(e\left(B_{j}\right)\right)\right| \leq 1$.

To finish the proof we define $A_{p}:=A \backslash\left(A_{1} \cup A_{2} \cup \cdots \cup A_{p-2} \cup A_{p-1}\right)$ and $\mu_{p}:=\mu_{p-1}$.

Lemma 3 Let $\mathscr{A}$ be an algebra of subsets of a set $\Omega, A \in \mathscr{A}$ and $M_{n}, n \in 1,2, \ldots, a$ weak*-closed and absolutely convex subset of ba( $\mathscr{A})$ such that for each finite subset $\mathrm{Q}$ of $\mathscr{A}$

$$
\sup _{\mu \in M_{n} \cap\{e(D): D \in \mathrm{Q}\}^{\circ}}|\mu|(A)=\infty
$$

for $n=n_{1}, n_{2}, \ldots, n_{p}$ and for an infinity of values of $n$. For each $\alpha \in \mathbb{R}^{+}$and each finite subset $\left\{B_{i}: 1 \leq i \leq n\right\}$ of $\mathscr{A}$ there exists in $A$ a partition $\left\{A_{1}, A \backslash A_{1}\right\} \in \mathscr{A} \times \mathscr{A}$ and $a$ measure $\mu_{1} \in M_{n_{1}}$ such that

$$
\left|\mu_{1}\left(e\left(A_{1}\right)\right)\right|>\alpha \text { and } \sum_{j=1}^{n}\left|\mu_{1}\left(e\left(B_{j}\right)\right)\right| \leq 1
$$

and for each finite subset $\mathrm{Q}$ of $\mathscr{A}$

$$
\sup _{\mu \in M_{n} \cap\{e(D): D \in \mathrm{Q}\}^{\circ}}|\mu|\left(A \backslash A_{1}\right)=\infty
$$

for $n=n_{1}, n_{2}, \ldots, n_{p}$ and for an infinity of values of $n$.

Proof By Lemma 2 for each $(p+2, \alpha) \in(\mathbb{N} \backslash\{0,1\}) \times \mathbb{R}^{+}$and for the subset $\left\{B_{i}: 1 \leq\right.$ $i \leq n\}$ of $\mathscr{A}$ there exists a partition $\left\{D_{i}: D_{i} \in \mathscr{A}, 1 \leq i \leq p+2\right\}$ of $A$ and a subset $\left\{v_{i}: 1 \leq i \leq p+2\right\}$ of $M_{n_{1}}$ such that

$$
\left|v_{i}\left(e\left(D_{i}\right)\right)\right|>\alpha \text { and } \sum_{j=1}^{n}\left|v_{i}\left(e\left(B_{j}\right)\right)\right| \leq 1 \text {, for } 1 \leq i \leq p+2 .
$$

From Claim 4 and for each $1 \leq j \leq p$ there exists $i_{j} \in\{1,2, \ldots, p+2\}$ such that for each finite subset $Q$ of $\mathscr{A}$

$$
\sup _{\mu \in M_{n_{j}} \cap\{e(D): D \in Q\}^{\circ}}|\mu|\left(D_{n_{i_{j}}}\right)=\infty
$$

and also there exists $i_{0} \in\{1,2, \ldots, p+2\}$ such that for each finite subset $Q$ of $\mathscr{A}$

$$
\sup _{\mu \in M_{n} \cap\{e(D): D \in Q\}^{\circ}}|\mu|\left(D_{n_{i_{0}}}\right)=\infty
$$

for infinite values of $n$. Let us suppose that $i^{*} \in\{1,2, \ldots, p+2\} \backslash\left\{i_{m}: m=0,1, \ldots, p\right\}$. To finish this proof let $\mu_{1}:=v_{i^{*}}$ and $A_{1}:=D_{i^{*}}$. Then

$$
\left|\mu_{1}\left(e\left(A_{1}\right)\right)\right|=\left|v_{i^{*}}\left(e\left(D_{i^{*}}\right)\right)\right|>\alpha \text { and } \sum_{j=1}^{n}\left|\mu_{1}\left(e\left(B_{j}\right)\right)\right|=\sum_{j=1}^{n}\left|v_{i}\left(e\left(B_{j}\right)\right)\right| \leq 1
$$

and for each finite subset $Q$ of $\mathscr{A}$

$$
\sup _{\mu \in M_{n} \cap\{e(D): D \in Q\}^{\circ}}|\mu|\left(A \backslash A_{1}\right)=\infty
$$

\section{照 Springer}


for $n=n_{1}, n_{2}, \ldots, n_{p}$ and for an infinity of values of $n$, because $A \backslash A_{1}=A \backslash D_{i^{*}}$ contains $\cup\left\{D_{n_{i_{j}}}: 0 \leq j \leq p\right\}$.

This Lemma may be applied without the finite subset $\left\{B_{i}: 1 \leq i \leq n\right\}$ of $\mathscr{A}$. Then we get that $\left|\mu_{1}\left(e\left(A_{1}\right)\right)\right|>\alpha$ and that for each finite subset $Q$ of $\mathscr{A}$ the set $M_{n} \cap\{e(D): D \in Q\}^{\circ}$ is unbounded in $\mathrm{ba}(\mathscr{A})$ for $n=n_{1}, n_{2}, \ldots, n_{p}$ and for an infinity of values of $n$.

Proposition 4 Let $\mathscr{A}$ be an algebra of subsets of a set $\Omega, A \in \mathscr{A}$ and $M_{n}, n \in 1,2, \ldots a$ weak*-closed and absolutely convex subset of ba $(\mathscr{A})$ such that for each finite subset $\mathrm{Q}$ of $\mathscr{A}$

$$
\sup _{\mu \in M_{n} \cap\{e(D): D \in \mathrm{Q}\}^{\circ}}|\mu|(A)=\infty
$$

for $n=n_{1}, n_{2}, \ldots, n_{p}$ and for an infinity of values of $n$. For each $(p, \alpha) \in(\mathbb{N} \backslash\{0,1\}) \times \mathbb{R}^{+}$ and each subset $\left\{B_{i}: 1 \leq i \leq n\right\}$ of $\mathscr{A}$ there exists a partition $\left\{A_{i}: A_{i} \in \mathscr{A}, 1 \leq i \leq p+1\right\}$ of $A$ and $\mu_{i} \in M_{n_{i}}, 1 \leq i \leq p$, such that

$$
\left|\mu_{i}\left(e\left(A_{i}\right)\right)\right|>\alpha, \sum_{j=1}^{n}\left|\mu_{i}\left(e\left(B_{j}\right)\right)\right| \leq 1, \text { for } 1 \leq i \leq p
$$

and for each finite subset $\mathrm{Q}$ of $\mathscr{A}$

$$
\sup _{\mu \in M_{n} \cap\{e(D): D \in \mathrm{Q}\}^{\circ}}|\mu|\left(A_{p+1}\right)=\infty
$$

for $n=n_{1}, n_{2}, \ldots, n_{p}$ and for an infinity of values of $n$.

Proof The Lemma 3 provides in $A$ a subset $A_{1} \in \mathscr{A}$ and $\mu_{1} \in M_{n_{1}}$ such that

$$
\left|\mu_{1}\left(e\left(A_{1}\right)\right)\right|>\alpha \text { and } \sum_{j=1}^{n}\left|\mu_{1}\left(e\left(B_{j}\right)\right)\right| \leq 1
$$

and for each finite subset $Q$ of $\mathscr{A}$

$$
\sup _{\mu \in M_{n} \cap\{e(D): D \in Q\}^{\circ}}|\mu|\left(A \backslash A_{1}\right)=\infty
$$

for $n=n_{1}, n_{2}, \ldots, n_{p}$ and for an infinity of values of $n$. If we apply again Lemma 3 to $A \backslash A_{1}$ we get $A_{2} \in \mathscr{A}, A_{2} \subset A \backslash A_{1}$, and $\mu_{2} \in M_{n_{2}}$ such that

$$
\left|\mu_{2}\left(e\left(A_{2}\right)\right)\right|>\alpha, \sum_{j=1}^{n}\left|\mu_{2}\left(e\left(B_{j}\right)\right)\right| \leq 1
$$

and for each finite subset $Q$ of $\mathscr{A}$

for $n=n_{1}, n_{2}, \ldots, n_{p}$ and for an infinity of values of $n$.

Following this method, for each $1 \leq i \leq p-1$ we get in $A$ the pairwise disjoint subsets $A_{i} \in \mathscr{A}$ and in $\mathrm{ba}(\mathscr{A})$ the measures $\mu_{i} \in M_{n_{i}}, 1 \leq i \leq p-1$, such that

$$
\left|\mu_{i}\left(e\left(A_{i}\right)\right)\right|>\alpha, \sum_{j=1}^{n}\left|\mu_{i}\left(e\left(B_{j}\right)\right)\right| \leq 1
$$


and for each finite subset $Q$ of $\mathscr{A}$

$$
\sup _{\mu \in M_{n} \cap\{e(D): D \in Q\}^{\circ}}|\mu|\left(A \backslash\left(A_{1} \cup A_{2} \cup \cdots \cup A_{p-1}\right)\right)=\infty
$$

for $n=n_{1}, n_{2}, \ldots, n_{p}$ and for an infinity of values of $n$. The Claim 3 applied to $A \backslash\left(A_{1} \cup\right.$ $\left.A_{2} \cup \cdots \cup A_{p-1}\right)$ provides $A_{p} \in \mathscr{A}, A_{p} \subset A \backslash\left(A_{1} \cup A_{2} \cup \cdots \cup A_{p-1}\right)$, and $\mu_{p} \in M_{n_{p}}$ such that

$$
\left|\mu_{i}\left(e\left(A_{p}\right)\right)\right|>\alpha, \sum_{j=1}^{n}\left|\mu_{i}\left(e\left(B_{j}\right)\right)\right| \leq 1
$$

and for each finite subset $Q$ of $\mathscr{A}$

$$
\sup _{\mu \in M_{n} \cap\{e(D): D \in Q\}^{\circ}}|\mu|\left(A \backslash\left(A_{1} \cup A_{2} \cup \cdots \cup A_{p-1} \cup A_{p}\right)\right)=\infty
$$

for $n=n_{1}, n_{2}, \ldots, n_{p}$ and for an infinity of values of $n$. With $A_{p+1}:=A \backslash\left(A_{1} \cup A_{2} \cup \cdots \cup\right.$ $\left.A_{p-1} \cup A_{p}\right)$ the proof is done.

Proposition 5 Let $\left\{\mathscr{B}_{n}: n \in \mathbb{N}\right\}$ be an increasing covering of a $\sigma$-algebra $\Sigma$ of subsets of a set $\Omega$. If $\mathscr{B}_{n}$ is not a Nikodým set for ba $(\Sigma)$ for each $n \in \mathbb{N}$ then for each $(i, j) \in \mathbb{N}^{2}$, such that $1 \leq i \leq j$, there exists $A_{i j} \in \Sigma$ and $\mu_{i j} \in \mathrm{ba}(\Sigma)$ such that the sets $A_{i j}$ are pairwise disjoint, for each natural number $i$ the set of measures $\left\{\mu_{i j}: j \in \mathbb{N}, j \geq i\right\}$ is pointwise bounded in $\mathscr{B}_{i}$ and

$$
\left|\mu_{i j}\left(e\left(A_{i j}\right)\right)\right|>j, \sum_{1 \leq k \leq m<j}\left|\mu_{i j}\left(e\left(A_{k m}\right)\right)\right| \leq 1 .
$$

Proof By Nikodým boundedness theorem $\Sigma$ is a Nikodým set for ba $(\Sigma)$, hence by Proposition 3 there exists $p \in \mathbb{N}$ such that for each $n \geq p$ there exists in ba $(\mathscr{A})$ an absolutely convex and weak*-closed subset $M_{n}$ that it is pointwise bounded in $\mathscr{B}_{n}$ and for each finite subset $Q$ of $\mathscr{A}$

$$
\sup _{\mu \in M_{n} \cap\{e(D): D \in Q\}^{\circ}}|\mu|(A)=\infty .
$$

Deleting the first $p-1$ sets $\mathscr{B}_{n}$ and renumbering the subindex $n$, I mean changing $n$ by $n-p+1$, we may suppose that $p=1$. The proof will be obtained by induction on $j$.

For $j=1$, the Lemma 3 with $\mathscr{A}=\Sigma, n=n_{1}=1$ and $\alpha=1$ provides a measure $\mu_{11} \in M_{n_{1}}$ and $A_{11} \in \Sigma$ such that

$$
\left|\mu_{11}\left(e\left(A_{11}\right)\right)\right|>1
$$

and for each finite subset $Q$ of $\Sigma$ we have that

$$
\sup _{\mu \in M_{n} \cap\{e(D): D \in Q\}^{\circ}}|\mu|\left(\Omega \backslash A_{11}\right)=\infty,
$$

for $n=n_{1}$ and for the elements $n$ of an infinity subset $N_{1}$ of $\mathbb{N} \backslash\left\{n_{1}\right\}$. Then let $n_{2}=\min \{n$ : $\left.n \in N_{1}\right\}$.

By Proposition 4 with $\mathscr{A}=\Sigma, A=\Omega \backslash A_{11}, n \in\left\{n_{1}, n_{2}\right\} \cup\left(N_{1} \backslash\left\{n_{2}\right\}\right), p=\alpha=2$ and with $\left\{B_{i}: 1 \leq i \leq n\right\}$ equal to $\left\{A_{11}\right\}$ we obtain two measures $\mu_{i 2} \in M_{n_{i}}, i=1,2$, and two disjoints elements of $\Sigma, A_{12}$ and $A_{22}$, contained in $\Omega \backslash A_{11}$ such that

$$
\left|\mu_{i 2}\left(e\left(A_{i 2}\right)\right)\right|>2,\left|\mu_{i 2}\left(e\left(A_{11}\right)\right)\right| \leq 1 \text {, for } 1 \leq i \leq 2
$$

\section{를 Springer}


and for each finite subset $Q$ of $\Sigma$ we have that

$$
\sup _{\mu \in M_{n} \cap\{e(D): D \in Q\}^{\circ}}|\mu|\left(\Omega \backslash\left(A_{11} \cup A_{12} \cup A_{22}\right)=\infty,\right.
$$

for $n \in\left\{n_{1}, n_{2}\right\} \cup N_{2}$, where $N_{2}$ is an infinite subset of $N_{1} \backslash\left\{n_{2}\right\}$. Then we define $n_{3}=$ $\min \left\{n: n \in N_{2}\right\}$.

Let's suppose that the step $j$ produces the measures $\mu_{i j} \in M_{n_{i}}$ and the pairwise disjoints elements $A_{i j}, 1 \leq i \leq j$, contained in $\Omega \backslash\left(\cup\left\{A_{k m}: 1 \leq k \leq m<j\right\}\right)$ with $A_{i j} \in \Sigma$ such that

$$
\left|\mu_{i j}\left(e\left(A_{i j}\right)\right)\right|>j, \sum_{1 \leq k \leq m<j}\left|\mu_{i j}\left(e\left(A_{k m}\right)\right)\right| \leq 1, \text { for } 1 \leq i \leq j
$$

and for each finite subset $Q$ of $\Sigma$ we have that

$$
\sup _{\mu \in M_{n} \cap\{e(D): D \in Q\}^{\circ}}|\mu|\left(\Omega \backslash\left(\cup\left\{A_{k m}: 1 \leq k \leq m \leq j\right\}\right)\right)=\infty
$$

for $n=\left\{n_{1}, n_{2}, \ldots, n_{j}\right\} \cup N_{j}$, with $N_{j}$ an infinity subset of $N_{j-1} \backslash\left\{n_{j}\right\}$.

Then we define $n_{j+1}=\min \left\{n: n \in N_{j}\right\}$ and from Proposition 4 with $\mathscr{A}=\Sigma, A=$ $\Omega \backslash\left(\cup\left\{A_{k m}: 1 \leq k \leq m \leq j\right\}\right), n \in\left\{n_{1}, n_{2}, \ldots, n_{j}, n_{j+1}\right\} \cup\left(N_{j} \backslash\left\{n_{j+1}\right\}, p=\alpha=j+1\right.$ and with $\left\{B_{i}: 1 \leq i \leq n\right\}$ equal to $\left\{A_{k m}: 1 \leq k \leq m \leq j\right\}$ we obtain the measures $\mu_{i, j+1} \in M_{n_{i}}$ and the pairwise disjoints elements $A_{i, j+1}$ of $\Sigma, 1 \leq i \leq j+1$, such that each $A_{i, j+1}$ is contained in $\Omega \backslash\left(\cup\left\{A_{k m}: 1 \leq k \leq m \leq j\right\}\right)$,

$$
\left|\mu_{i, j+1}\left(e\left(A_{i, j+1}\right)\right)\right|>j+1, \sum_{1 \leq k \leq m<j+1}\left|\mu_{i, j+1}\left(e\left(A_{k m}\right)\right)\right| \leq 1, \text { for } 1 \leq i \leq j+1
$$

and for each finite subset $Q$ of $\Sigma$ we have that

$$
\sup _{\mu \in M_{n} \cap\{e(D): D \in Q\}^{\circ}}|\mu|\left(\Omega \backslash\left(\cup\left\{A_{k m}: 1 \leq k \leq m \leq j+1\right\}\right)\right)=\infty,
$$

for $n=\left\{n_{1}, n_{2}, \ldots, n_{j}, n_{j+1}\right\} \cup N_{j+1}$, where $N_{j+1}$ is an infinity subset $N_{j} \backslash\left\{n_{j+1}\right\}$. To finish the induction we define $n_{j+2}=\min \left\{n: n \in N_{j+1}\right\}$.

Theorem 2 Let $\left\{\mathscr{B}_{n}: n \in \mathbb{N}\right\}$ be an increasing covering of a $\sigma$-algebra $\Sigma$ of subsets of a set $\Omega$. There exists a $q \in \mathbb{N}$ such that $\mathscr{B}_{n}$ is a Nikodým set for ba $(\Sigma)$ for each $n \geq q$.

Proof Let's proceed by contradiction and suppose that every $\mathscr{B}_{n}$ is not a Nikodým set for ba $(\Sigma)$. By Proposition 5 for each $(i, j) \in \mathbb{N}^{2}$, such that $1 \leq i \leq j$, there exists $A_{i j} \in \Sigma$ and $\mu_{i j} \in \operatorname{ba}(\Sigma)$ such that

$$
\left|\mu_{i j}\left(e\left(A_{i j}\right)\right)\right|>j, \sum_{1 \leq k \leq m<j}\left|\mu_{i j}\left(e\left(A_{k m}\right)\right)\right| \leq 1,
$$

the sets $A_{i j}$ are pairwise disjoint and the set of measures $\left\{\mu_{i j}: j \in \mathbb{N}, j \geq i\right\}$ is pointwise bounded in $\mathscr{B}_{i}$, for each $i \in \mathbb{N}$.

We claim that there exists a sequence $\left(i_{n}, j_{n}\right)_{n \in \mathbb{N}}$ such that $\left(i_{n}\right)_{n \in \mathbb{N}}$ is the sequence of the first components of the sequence obtained when the elements of $\mathbb{N}^{2}$ are ordered by the diagonal order, i.e.,

$$
\left(i_{1}, i_{2}, i_{3}, i_{4}, i_{5}, i_{6}, i_{7}, \ldots\right)=(1,1,2,1,2,3,1, \ldots),
$$

and $\left(j_{n}\right)_{n \in \mathbb{N}}$ is a strict increasing sequence such that for each $n \in \mathbb{N}$

$$
\left|\mu_{i_{n}, j_{n}}\right|\left(\cup\left\{A_{i_{m}, j_{m}}: m>n\right\}\right) \leq 1 .
$$


Let $\left(i_{1}, j_{1}\right):=(1,1)$, suppose that $\left|\mu_{i_{1}, j_{1}}\right| \leq k_{1}$ and split the set $\{j \in \mathbb{N}: j>1\}$ in $k_{1}$ infinite subsets $N_{11}, \ldots, N_{1 k_{1}}$. At least one of this subsets, named $N_{1}$, verifies that

$$
\left|\mu_{i_{1}, j_{1}}\right|\left(\cup\left\{A_{i, j}: i \leq j, j \in N_{1}\right\}\right) \leq 1,
$$

because

$$
k_{1} \geq\left|\mu_{i_{1}, j_{1}}\right|=\sum_{1 \leq r \leq k_{1}}\left|\mu_{i_{1}, j_{1}}\right|\left(\cup\left\{A_{i, j}: i \leq j, j \in N_{1 r}\right\}\right) .
$$

Then we define $j_{2}:=\inf \left\{n: n \in N_{1}\right\}$. Suppose that we have obtained the natural number $j_{n}$ and the infinite subset $N_{n}$ of $\mathbb{N}$ such that

$$
\left|\mu_{i_{n}, j_{n}}\right|\left(\cup\left\{A_{i, j}: i \leq j, j \in N_{n}\right\}\right) \leq 1 .
$$

Then we define $j_{n+1}=\inf \left\{n: n \in N_{n}\right\}$ and if $\left|\mu_{i_{n+1}, j_{n+1}}\right| \leq k_{n+1}$ we split the set $\{j \in$ $\left.N_{n}: j>j_{n+1}\right\}$ in $k_{n+1}$ infinite subsets $N_{n+1,1}, \ldots, N_{n+1, k_{n+1}}$. At least one of this subsets, named $N_{n+1}$ verifies that

$$
\left|\mu_{i_{n+1}, j_{n+1}}\right|\left(\cup\left\{A_{i, j}: i \leq j, j \in N_{n+1}\right\}\right) \leq 1
$$

because

$$
k_{n+1} \geq\left|\mu_{i_{n+1}, j_{n+1}}\right|=\sum_{1 \leq r \leq k_{n+1}}\left|\mu_{i_{n+1}, j_{n+1}}\right|\left(\cup\left\{A_{i, j}: i \leq j, j \in N_{n+1, r}\right\}\right) .
$$

As $A=\cup\left\{A_{i_{m}, j_{m}}: m \in \mathbb{N}\right\} \in \Sigma$ there exists $r \in \mathbb{N}$ such that $A \in \mathscr{B}_{r}$. By construction, there exists and increasing sequence $\left(m_{s}: s \in \mathbb{N}\right)$ such that each $i_{m_{s}}=r, s \in \mathbb{N}$. Therefore the set of measures $\left\{\mu_{i_{m_{s}}}, j_{m_{s}}: s \in \mathbb{N}\right\}=\left\{\mu_{r, j_{m_{s}}}: s \in \mathbb{N}\right\}$ is pointwise bounded in $\mathscr{B}_{r}$ and, in particular

$$
\sup \left\{\left|\mu_{i_{m_{s}}, j_{m_{s}}}(A)\right|: s \in \mathbb{N}\right\}=\sup \left\{\left|\mu_{r, j_{m_{s}}}(A)\right|: s \in \mathbb{N}\right\}<\infty .
$$

But from

$$
\begin{aligned}
\left|\mu_{i_{m_{s}}, j_{m_{s}}}(A)\right|= & \left|\mu_{i_{m_{s}}, j_{m_{s}}}\left(\bigcup_{m \in \mathbb{N}} A_{i_{m}, j_{m}}\right)\right| \\
\geq & \left|\mu_{i_{m_{s}}, j_{m_{s}}}\left(A_{i_{m_{s}}, j_{m_{s}}}\right)\right|-\sum_{1 \leq k \leq m<j_{m_{s}}}\left|\mu_{i_{m_{s}}, j_{m_{s}}}\left(A_{k m}\right)\right| \\
& -\left|\mu_{i_{m_{s}}, j_{m_{s}}}\right|\left(\bigcup_{m>j_{m_{s}}} A_{i_{m}, j_{m}}\right)>j_{m_{s}}-2
\end{aligned}
$$

we get that $\lim _{s \rightarrow \infty}\left|\mu_{i_{m_{s}}, j_{m_{s}}}(A)\right|=\infty$, in contradiction with (10).

A proof of the web Nikodým property of every $\sigma$-algebra is presented in [11, Theorem 1]. It depends of properties of some families of subsets of $\cup\left\{\mathbb{N}^{p}: p \in \mathbb{N}\right\}$, called $N V$-trees in honor of Nikodým and Valdivia.

Problem 2 To get a proof of the property that every $\sigma$-algebra has web Nikodým property with basic results of Measure theory and Banach spaces.

Problem 3 Let $\mathscr{A}$ be an algebra of subsets of a set $\Omega$ such that $\mathscr{A}$ is a Nikodým set for $\mathrm{ba}(\mathscr{A})$. Is it true that $\mathscr{A}$ is a web Nikodým set for ba $(\mathscr{A})$.

This Problem is the web Nikodým version of [17, Problem 1].

\section{照 Springer}




\section{Conclusions}

We have proved that if $\left(\mathscr{B}_{m}\right)_{m=1}^{\infty}$ is an increasing covering of an algebra $\mathscr{A}$ that has $(V H S)$ property and there exist a $\mathscr{B}_{n}$ which is a Nikodým set for $b a(\mathscr{A})$ then there exists $\mathscr{B}_{q}$, with $q \geq p$, such that $\mathscr{B}_{q}$ has $(V H S)$ property, being this property defined in a natural way with the properties that define the $(V H S)$ property in an algebra. An increasing web of a $\sigma$-algebra $\Sigma$ contains an increasing web formed by sets that have (VHS) property and, in particular, if $\left(\mathscr{B}_{m}\right)_{m=1}^{\infty}$ is an increasing covering of a $\sigma$-algebra there exists $\mathscr{B}_{q}$ that has ( $V H S$ ) property. We do not know if this property holds for an algebra and we have proved that this problem is equivalent to the analogous Valdivia open problem for Nikodým property. Other two related open problems are proposed.

As a help to solve this aforementioned Valdivia problem we give a proof of Valdivia theorem stating that for each $\sigma$-algebra $\Sigma$ the set $\Sigma$ is a strong Nikodým set for $b a(\Sigma)$. This proof follows the scheme given by Valdivia in [16], but it is independent of the Barrelled spaces theory and it only needs basic results of Measure theory and Banach spaces.

Acknowledgements The author thanks both referees for valuable comments and suggestions.

\section{References}

1. Diestel, J.: Sequences and Series in Banach Spaces. Number 92 in Graduate Texts in Mathematics. Springer, New York (1984)

2. Diestel, J., Uhl, J.J.: Vector Measures. Number 15 in Mathematical Surveys and Monographs. American Mathematical Society, Providence (1977)

3. Ferrando, J.C.: Strong barrelledness properties in certain $l_{0}^{\infty}(\mathscr{A})$ spaces. J. Math. Anal. Appl. 190, 194202 (1995)

4. Ferrando, J.C., López-Alfonso, S., López-Pellicer, M.: On Nikodým and Rainwater sets for ba $(R)$ and a problem of M. Valdivia. Filomat 33(8), 2409-2416 (2019)

5. Ferrando, J.C., López-Pellicer, M.: Strong barrelledness properties in $l_{0}^{\infty}(x, \mathscr{A})$ and bounded finite additive measures. J. Math. Anal. Appl. 287, 727-736 (1990)

6. Ferrando, J.C., López-Pellicer, M., Sánchez Ruiz, L.M.: Metrizable Barrelled Spaces. Number 332 in Pitman Research Notes in Mathematics Series. Longman, Harlow (1995)

7. Ferrando, J.C., Sánchez Ruiz, L.M.: A survey on recent advances on the Nikodým boundedness theorem and spaces of simple functions. Rocky Mountain J. Math. 34, 139-172 (2004)

8. Kakol, J., López-Pellicer, M.: On Valdivia strong version of Nikodým boundedness property. J. Math. Anal. Appl. 446, 1-17 (2017)

9. Köthe, G.: Topological Vector Spaces I and II. Springer, Berlin (1979)

10. López-Alfonso, S.: On Schachermayer and Valdivia results in algebras of Jordan measurable sets. Rev. R. Acad. Cienc. Exactas Fís. Nat. Ser. A Mat. RACSAM 110, 799-808 (2016)

11. López-Alfonso, S., Mas, J., Moll, S.: Nikodým boundedness property for webs in $\sigma$-algebras. Rev. R. Acad. Cienc. Exactas Fís. Nat. Ser. A Mat. RACSAM 110, 711-722 (2016)

12. López-Alfonso, S., Moll, S.: The uniform bounded deciding property and the separable quotient problem. Rev. R. Acad. Cienc. Exactas Fís. Nat. Ser. A Mat. RACSAM 113, 1223-1230 (2019)

13. Pérez Carreras, P., Bonet, J.: Barrelled Locally Convex Spaces. Number 131 in North-Holland Mathematics Studies. Notas de Matemática. North-Holland Publishing Co., Amsterdam (1987)

14. López-Pellicer, M.: Webs and bounded finitely additive measures. J. Math. Anal. Appl. 210, 257-267 (1997)

15. Schachermayer, W.: On some classical measure-theoretic theorems for non-sigma-complete Boolean algebras. Dissertationes Math. (Rozprawy Mat.) 214, 33 (1982)

16. Valdivia, M.: On certain barrelled normed spaces. Ann. Inst. Fourier (Grenoble) 29, 39-56 (1979)

17. Valdivia, M.: On Nikodým boundedness property. Rev. R. Acad. Cienc. Exactas Fís. Nat. Ser. A Mat. RACSAM 107, 355-372 (2013)

Publisher's Note Springer Nature remains neutral with regard to jurisdictional claims in published maps and institutional affiliations. 